\title{
Falx Meningeoma Induced Optic Neuropathy-Clinical Manifestation and Differential Diagnosis: A Case Report
}

\author{
Skevas Christos", Görsch Isabel Carolin, Richard Gisbert, Linke Johannes Stephan \\ Universitätsklinikum Hamburg-Eppendorf, Klinik und Poliklinik für Augenheilkunde, Hamburg, Germany. \\ Email: \#c.skevas@uke.uni-hamburg.de
}

Received March 25 ${ }^{\text {th }}, 2013$; revised April 26 ${ }^{\text {th }}, 2013$; accepted June $20^{\text {th }}, 2013$

Copyright (C) 2013 Skevas Christos et al. This is an open access article distributed under the Creative Commons Attribution License, which permits unrestricted use, distribution, and reproduction in any medium, provided the original work is properly cited.

\begin{abstract}
Purpose: Meningiomas are the most frequently diagnosed neoplasms. Ocular symptoms vary depending on size and localisation of the tumor, which is capable of compressing critical anatomic structures. Patients and Methods: A 44-year-old immunocompetent male was admitted to our hospital for further investigation of his bilateral optic disc swelling. A complete ophthalmological examination was performed including visual field assessment (VF), visual evoked potentials (VEP), fluorescein angiography (FLA) and magnetic resonance imaging (MRI). The patient had experienced only minor visual disturbances. Serological testing was performed. Results: Serological testing showed positive IgM and IgGtiters for cytomegalovirus (CMV) and subsequent imaging (MRI) revealed a frontal falxcerebri meningioma. Conclusions: We present a case of falx meningioma with overlapping serologically active CMV in an otherwise healthy and immunocompetent individual. Detailed differential diagnostic workup in immunocompetent individuals presenting with clinically unremarkable bilateral disc swelling is recommended.
\end{abstract}

Keywords: Falx Meningeoma; Optic Neuropathy; Cytomegalovirus (CMV); Visual Disturbances; Fluorescein Angiography (FLA); Magnetic Resonance Imaging (MRI); Visual Evoked Potentials (VEP)

\section{Introduction}

Meningiomas are the most frequently diagnosed neoplasms accounting for $33.8 \%$ of all brain and central nervous system tumors reported in the USA between 2002 and 2006 [1]. Cushing defined falx meningioma as a tumor arising from the falxcerebri and concealed by the overlying cortex [2].

Ocular symptoms vary depending on size and localisation of the tumor, which is capable of compressing critical anatomic structures. Visual field abnormalities, optic atrophy, optic disc edema, diplopia and proptosis can be presented. Intracranial hypertension is defined as an increase in the intracranial pressure $>20 \mathrm{mmHg}$. Typical manifestations are headaches, vomiting and visual symptoms (transient visual obscurations, loss of visual acuity) [3]. We report on a 44-year-old immunocompetent male with bilateral swelling of the optic discs due to falxmeningeoma. The diagnosis was overlapped by positive IgM and IgGtiters for CMV.

\footnotetext{
*Disclosure declaration: There are no proprietary interests and no financial support was received. No conflicts of interest regarding this article exist.

${ }^{\#}$ Corresponding author.
}

\section{Materials and Methods}

A 44-year-old male with unremarkable medical history presented in our clinic with a bilateral optic disc swelling, which was diagnosed during a routine ophthalmological examination. Patient was not taking any medication and denied having any reduced visual acuity, transient visual disturbances or headaches. A complete ophthalmological examination was performed including corrected distance visual acuity (CDVA), visual field (VF) assessment, fundoscopy, visual evoked potentials (VEP), fluorescein angiography (FLA), serological examinations and magnetic resonance imaging (MRI).

\section{Results}

Corrected distance visual acuity (CDVA) was 20/20 for both eyes $(\mathrm{R}-4.5$ sph -0.75 cyl A 83 and $\mathrm{L}-4.5 \mathrm{sph}$ -1.25 cyl A 177) but visual field (VF) assessment (Humphrey Visual Field Analyzer, protocol 24-2; Carl Zeiss Ophthalmic Systems Inc., Dublin, CA) showed bilateral $(\mathrm{R}>\mathrm{L})$ inferior altitudinal field defects. No afferent pupillary defect or colour desaturation was evident. Anterior chamber assessment showed no pathology, and fun- 
doscopy confirmed a bilateral $(\mathrm{R}>\mathrm{L})$ optic disc edema without any signs of retinal haemorrhages and no cells in the hyaloidbody. Visual evoked potentials (VEP) were within normal limits and fluorescein angiography (FLA) showed a late phase disc staining. Ultrasonography of the optic nerve sheath, Doppler ultrasonography and colordoppler imaging (CDI) elicited no abnormalities. Routine and differential blood counts, C-reactive protein (CRP), protein $\mathrm{C}$ and $\mathrm{S}$ deficiency, APC resistance, Factor V Leiden, anti-cardiolipin antibodies were all within normal range. Further infectious serologic blood tests (Herpes Simplex virus, Varizella Zoster virus, Cytomegalovirus, Ebstein-Barr virus, Lues, Toxoplasmosis, Borreliosis, HIV) were performed and revealed positive IgG and IgMtiters for Cytomegalovirus (CMV) indicative for an active CMV infection. The ensuing physical, neurological as well as chest X-ray examinations were unremarkable with no signs of splenomegaly. A 2-week course of intravenous ganciclovir $(2 \times 5 \mathrm{mg}$ pro $\mathrm{Kg}$ i.v $)$ treatment was commenced and additionally a cerebral imaging (MR) was performed. A $4.0 \mathrm{~cm} \times 4.5 \mathrm{~cm} \times 4.0$ $\mathrm{cm}$ solitary, well defined, parasagittal frontal meningioma (Figures 1 and 2) originating from falxcerebri was diagnosed. A surgical resection of the tumor had been scheduled after consultation with the department of neurosurgery.

\section{Discussion}

Among the differential diagnosis of bilateral optic disc edema, intracranial hypertension and infectious papillitis are most frequently reported. The differentiation can fur-

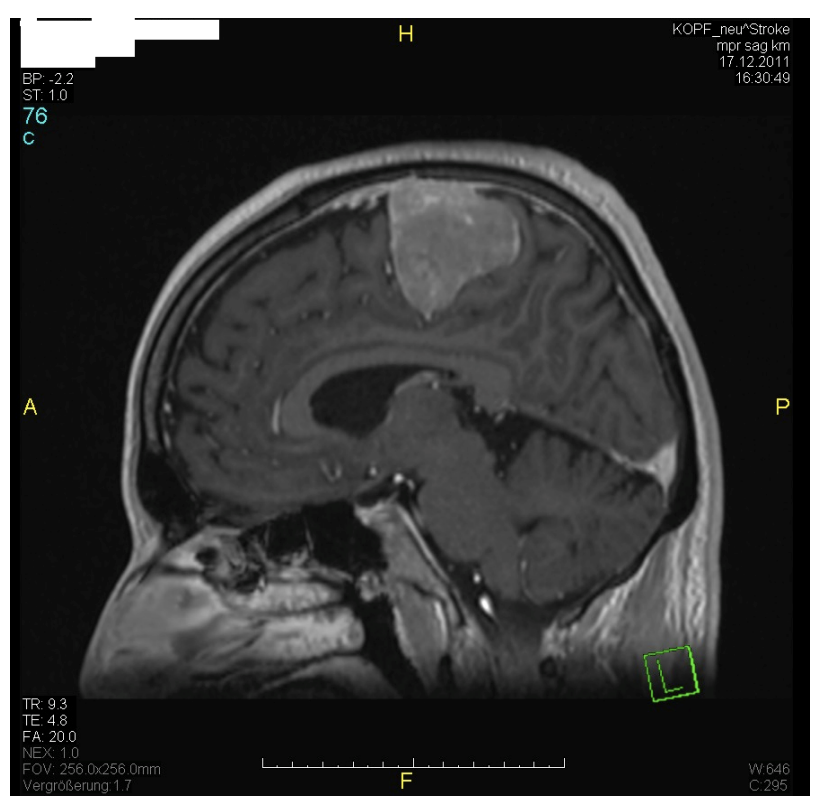

Figure 1. A $4.0 \mathrm{~cm} \times 4.5 \mathrm{~cm} \times 4.0 \mathrm{~cm}$ solitary, well defined, parasagittal frontal meningioma originating from falxcerebri.

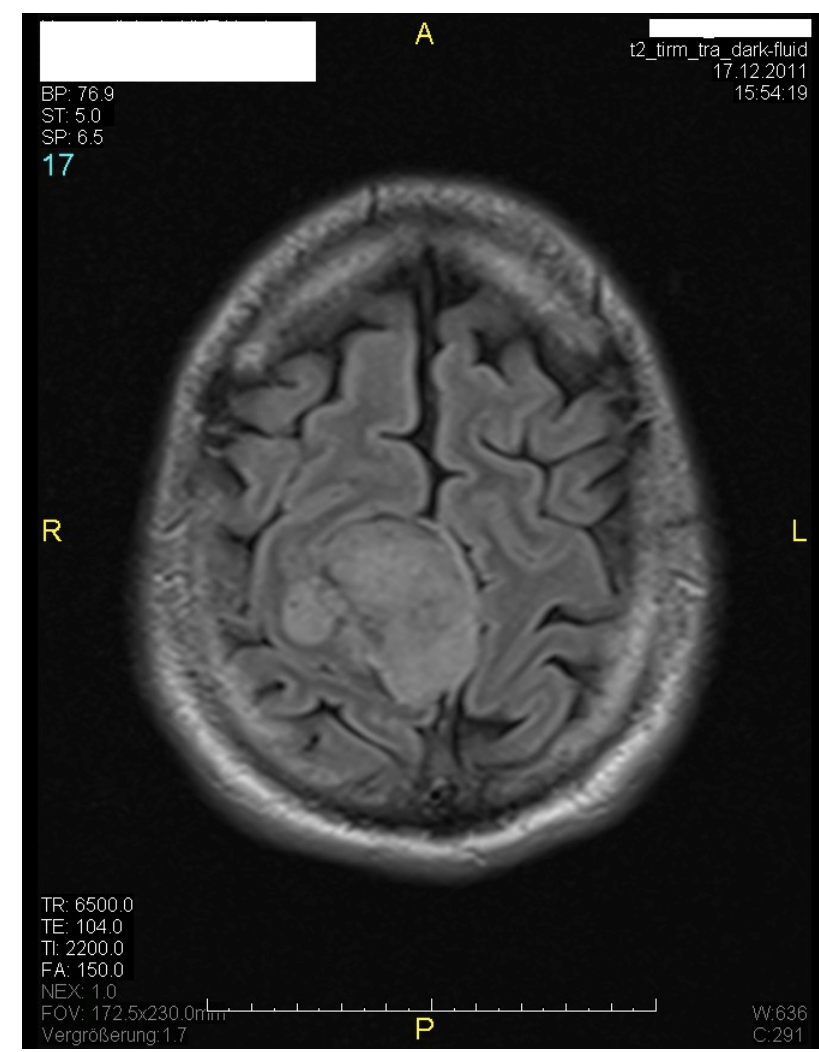

Figure 2. A $4.0 \mathrm{~cm} \times 4.5 \mathrm{~cm} \times 4.0 \mathrm{~cm}$ solitary, well defined, parasagittal frontal meningioma originating from falxcerebri.

ther be hampered by the absence of neurological symptoms. Classification of meningiomas according to Cushing and Eisenhardt was based on surgical findings of a dural or bony site of origin and depending on their origin in the falx, they can be divided into anterior, middle or posterior types [2]. Optic disc edema which is fortuitously discovered in patients without complaints can insidiously lead to visual handicap, due to chronic optic disc edema and optic atrophy. One feature of optic disc edema from raised $\mathrm{ICH}$ is that normal vision usually continues without interference for many weeks or even months. Hayreh speculated that the cause of the laminar transport block is mechanical and the visual loss in chronic optic disc edema results from ischaemia secondary to optic disc swelling [4].

Cytomegalovirus (CMV) is a neurotropic virus with a predilection to the retina and the central nervous system. The natural course of CMV papillitis leads to blindness. Grossniklaus et al. detected CMV inflammation cell infiltrated in the retrolaminar portion of the optic nerve. The pathological examination shows signs of necrosis [5]. CMV retinitis usually affects individuals that have been already diagnosed with acquired immunodeficiency syndrome (AIDS) (stages of advanced immunsupression-CD $4+$ counts of $<50$ cells $/ \mathrm{mm}^{3}$ ) [6]. The therapy of CMV 
retinitis/optic neuritis consists of intravenous ganciclovir or intravenous foscarnet, but their use in combination is also presented. The combination is more effective in controlling progression of CMV retinitis in relapsed patients than monotherapy alone $[7,8]$. Besides patients with immune deficiency (AIDS), there are reports of CMV induced optic neuropathy in healthy immunocompetent individuals $[9,10]$.

We present to the best of our knowledge the first case of falx meningioma with overlapping serologically active CMV in an otherwise healthy and immunocompetent individual. A step by step approach for detailed differential diagnostic workup in immunocompetent individuals presenting with clinically unremarkable bilateral disc swelling is recommended. Treatment should involve a harmonious cooperative effort between the ophthalmologist and physicians of other specialities such as microbiology, neurology and neurosurgery.

\section{REFERENCES}

[1] CBTRUS, "Statistical Report: Primary Brain and Central Nervous System Tumors Diagnosed in Eighteen States in 2002-2006," Central Brain Tumor Registry of the United States, Hinsdale, 2009-2010.

[2] H. Cushing and L. Eisenhardt, "Meningiomas. Their Classification, Regional Behavior, Life History and Surgical End Results," Bulletin of the Medical Library Association, Vol. 27, No. 2, 1938, pp. 224-249.

[3] D. I. Friedman and D. M. Jacobson, "Diagnostic Criteria for Idiopathic Intracranial Hypertension," Neurology, Vol.
59, No. 10, 2002, pp. 1492-1495.

[4] S. S. Hayreh, "Optic Disc Edema in Raised Intracranial Pressure. VI. Associated Visual Disturbances and Their Pathogenesis," Archives of Ophthalmology, Vol. 95, No. 9, 1977, pp. 1566-1579. doi:10.1001/archopht.1977.04450090088007

[5] H. E. Grossniklaus, K. E. Frank and R. L. Tomsak, "Cytomegalovirus Retinitis and Optic Neuritis in Acquired Immune Deficiency Syndrome: Report of a Case," Ophthalmology, Vol. 94, No. 12, 1987, pp. 1601-1604.

[6] J. D. Roarty, E. J. Fisher and J. J. Nussbaum, "Long-Term Visual Morbidity of Cytomegalovirus Retinitis in Patients with Acquired Immune Deficiency Syndrome," Ophthalmology, Vol. 100, No. 11, 1993, pp. 1685-1688.

[7] D. N. Friedberg, "Cytomegalovirus Retinitis: Diagnosis and Status of Systemic Therapy," Journal of Acquired Immune Deficiency Syndromes \& Human Retrovirology, Vol. 14, No. 1, 1997, pp. S1-S6. doi:10.1097/00042560-199700001-00002

[8] A. M. Mansor and H. K. Li, "Cytomegalovirus Optic Neuritis: Characteristics, Therapy and Survival," Ophthalmologica, Vol. 209, No. 5, 1995, pp. 260-266. doi: $10.1159 / 000310627$

[9] E. Baglivo, P. M. Leuenberger and K. H. Krause, "Presumed Bilateral Cytomegalovirus-Induced Optic Neuropathy in an Immunocompetent Person: A Case Report," Journal of Neuro-Ophthalmology, Vol. 16, No. 1, 1996, pp. 14-17. doi:10.1097/00041327-199603000-00004

[10] S. R. De Silva, G. Chohan, D. Jones and M. Hu, "Cytomegalovirus Papillitis in an Immunocompetent Patient," Journal of Neuro-Ophthalmology, Vol. 28, No. 2, 2008, pp. 126-127. doi:10.1097/WNO.0b013e3181782fed 